\title{
A Comprehensive Analytical Dynamic Model of a T-Beam
}

\author{
Andrew J. Hull \\ Undersea Warfare Weapons, Vehicles, and Defensive Systems Department, Naval Undersea Warfare Centre, New- \\ port, Rhode Island 02841 USA.
}

\section{Daniel Perez and Donald L. Cox}

Sensors and Sonar Systems Department, Naval Undersea Warfare Centre, Newport, Rhode Island 02841 USA.

(Received 3 May 2017; accepted 26 February 2018)

\begin{abstract}
This paper derives a comprehensive analytical dynamic model of a T-shaped beam that includes in-plane and outof-plane vibrations for mid-frequency range analysis, defined here as approximately $1 \mathrm{kHz}$ to $10 \mathrm{kHz}$. The web, right part of the flange, and left part of the flange of the T-beam are modelled independently with two-dimensional elasticity equations for the in-plane motion and the classical flexural plate equation for the out-of-plane motion. The differential equations are solved with unknown wave propagation coefficients multiplied by circular spatial domain functions, which are inserted into equilibrium and continuity equations at the intersection of the web and flange and into boundary conditions at the edges of the system resulting in 24 algebraic equations. These equations are solved to yield the wave propagation coefficients and this produces a solution to the displacement field in all three dimensions. An example problem is formulated and compared to solutions from Bickford beam theory and finite element analysis. Higher order branch waves are discussed and a simplified symmetric model is presented.
\end{abstract}

\section{INTRODUCTION}

Beams have numerous applications in the aerospace, maritime, automotive, building, and mining industries. They are typically structural members and are designed to resist an external force either applied directly or applied to a body that they are supporting. They provide a concentrated stiffness at one or more locations in a mechanical system that has various design objectives. There is an extremely large body of analytical and experimental papers that model and analyse various types of beams. The first equation of motion of a beam was developed by Bernoulli and Euler and this equation is presented in almost every text on mechanical vibrations. ${ }^{1}$ This theory uses the assumption that all sections rotate orthogonal to the neutral axis of the beam. Timoshenko revised this equation so that the rotation angle was a function of the polar inertia of the beam. ${ }^{2}$ Beam theory has been made more accurate by the inclusion of higher order displacement functions, usually in the axial direction. Bickford used a third-order polynomial through the thickness of the beam to model the in-plane displacement field. ${ }^{3}$ Karama et al. used an exponential function to model the shear distribution in the beam. ${ }^{4}$ Other functional distributions are possible and have also been similarly applied to plate theory. 5,6

Over the years, beams have become more geometrically diverse and their shapes have been modified from rectangular to L-, T-, I-, and H-shaped, channel-shaped, and hollow box designs. Park et al. studied longitudinal wave motion of finite coupled thin plates. ${ }^{7}$ Kessissoglou added the flexural motion to the previous reference so that a full three-dimensional analysis of an L-shaped finite plate became possible. ${ }^{8}$ Du et al. investigated free vibration of coupled rectangular plates with general boundary conditions, using energy methods to derive the dif-

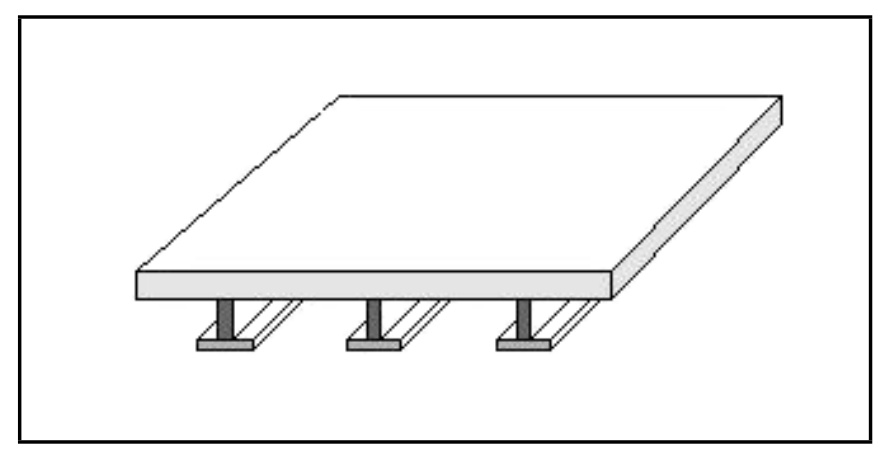

Figure 1. Three inverted T-beams supporting a plate.

ferential equations of motion. ${ }^{9}$ Chen et al. analysed vibrations in box type plate structures. ${ }^{10}$ Wang addressed the problem of finite coupled plates whose intersection contained a mass. ${ }^{11}$ T-shaped beams have had some investigations, generally applied to concrete or reinforced concrete structures where static analysis and ultimate strength are the major focal points of the research. ${ }^{12,13}$ Langley and Heron derived a method to calculate wave transmission coefficients of plate and beam junctions. ${ }^{14}$ Keir et al. investigated coupled rectangular plates with an emphasis on the effect of active control of these systems. ${ }^{15}$ Mitrou et al. researched wave transmission in two-dimensional structures using a mixed finite element and wave and finite element methods. ${ }^{16}$

Beam reinforced structures have been analysed for many years. A typical example is shown in Fig. 1. Fluid-loaded stiffened plates have been researched using flexural wave plate models by Mace ${ }^{17,18}$ and Lin and Hayek ${ }^{19}$ with a general emphasis on structural acoustic responses. The plate governing equations in a stiffened plate analysis has been extended to admit fully elastic wave propagation by Hull and Welch and this 


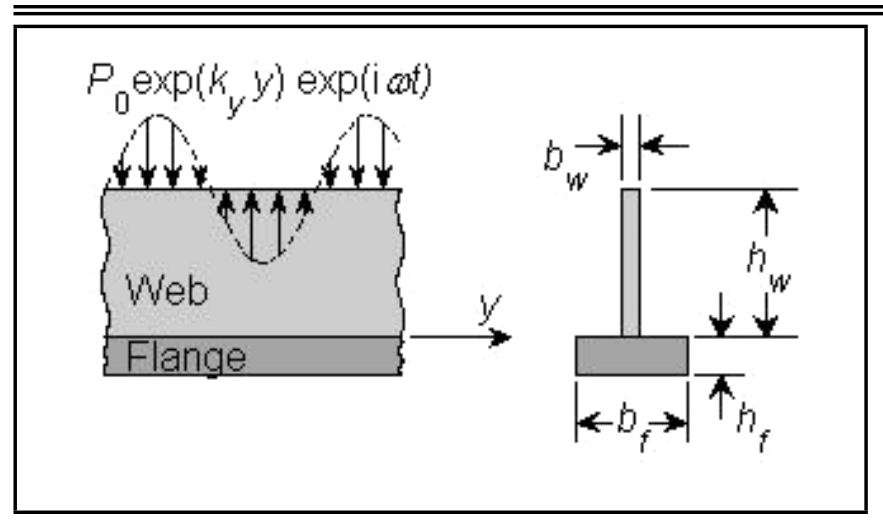

Figure 2. Inverted T-beam with dimensions and a normal pressure excitation.

allows much higher frequency studies compared to previous flexural wave models. ${ }^{20}$ Models of these structures typically have an infinite length assumption as the energy propagates down the length of the structure and due to the large spatial lengths and slight damping present does not form a standing wave pattern. The model of the beam reinforcement has typically been an infinite length Bernoulli-Euler or Timoshenko model which has low- to mid-frequency range limitations.

This paper develops an analytical model of a T-shaped beam for mid-frequency range analysis. In this model, the web and flange of the T-beam are modelled independently with twodimensional elasticity accounting for the in-plane motion and the classical flexural plate equation accounting for the outof-plane motion. The method presented here combines six in-plane components and three out-of-plane components into a single model that can predict the response of a T-beam to various external loads. The web and flange are joined by twelve constraint equations and the free and forced edges are modelled with twelve additional force and moment equations. Modelling the beam in this manner allows the web and flange equations of motion to incorporate coupled in-plane and outof-plane plate responses, and these equations make the model much more accurate compared to previous shear deformation models. ${ }^{4,5}$ For the specific example presented in this paper, the three-dimensional displacement fields are studied with respect to independent loads in the three primary axes of the Cartesian coordinate system. Model comparisons are made to the Bickford beam model and a finite element model. It is shown that for the specific example presented here, the accurate frequency region of the normal displacement divided by normal pressure is increased from $1 \mathrm{kHz}$ of the Bickford beam model to about $8 \mathrm{kHz}$ for the new beam model. The new model admits higher order branch wave propagation modes and the locations of these modes in the wavenumber-frequency plane are analytically calculated.

\section{SYSTEM MODEL}

The system under consideration is an inverted T-beam with continuous spatial and time harmonic excitation on the top of the structure. A schematic of this system is shown in Fig. 2. The narrow top portion is called the web and the wide lower portion is called the flange. Usually this type of beam is symmetric about the mid plane of the web, but this is not a necessary condition for the model, as the model presented in this

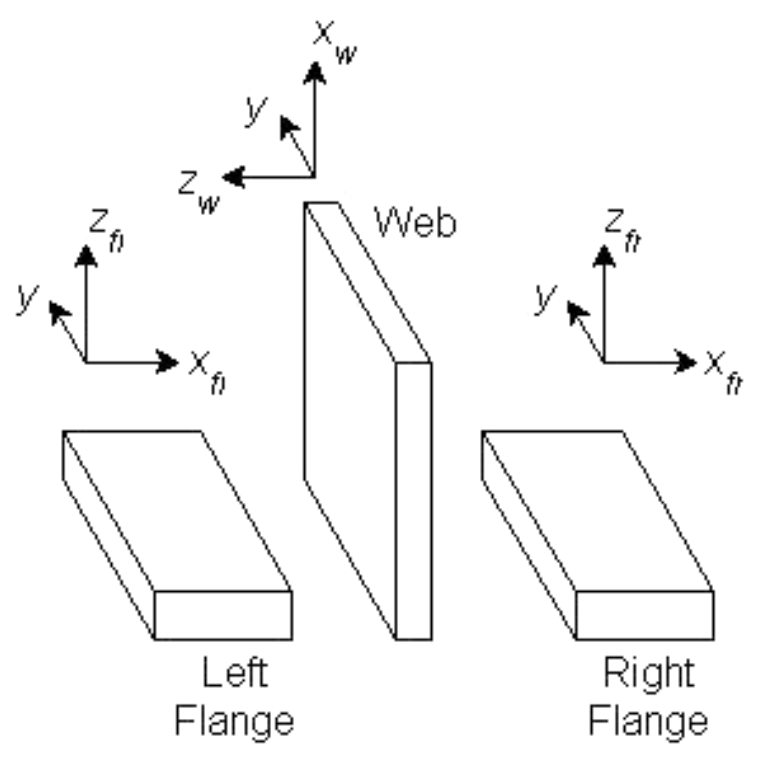

Figure 3. Diagram of T-beam with component coordinate systems.

section will capture the dynamics of an asymmetric beam. The problem is analytically modelled using the two-dimensional plane stress elastic equations for in-plane motions of the web and the flange and classical plate equations for the out-of-plane motion of the web and the flange. This model is an extension of a previous model, where two finite plates were oriented in an L-shaped configuration and the power transmission of the structure caused by transverse loading on one of the plates was studied. ${ }^{8}$ The model derived here varies from the previous model in that it is an infinite beam, so that the differential equations and boundary conditions admit different functions than that of a finite plate, a flange has been added to make it a T-configuration rather than an L-configuration and loading and analysis are in all three directions of the coordinate system, so that the beam is excited by normal pressure, axial pressure, and transverse pressure, all represented by continuous analytical functions.

The model uses the following assumptions: (1) the system has infinite spatial extent in the $y$-direction, (2) the excitation is at a fixed frequency and fixed wavenumber in the $y$ direction, (3) the angle at the intersection of the web and the flange is always a right angle, (4) the material properties of the web and flange are identical, and (5) the particle motion is linear. The model is developed by analysing the system as three separate components: the web, the left part of the flange, and the right part of the flange. For all three components, the two-dimensional elasticity equations of motion are used for the in-plane motion and classical plate equations are used for out-of-plane motion. The equations modelling the in-plane motion of the web and flange begin with the Navier-Cauchy fully elastic equations of motion. ${ }^{21}$ These are reduced to twodimensional plane stress equations of motion, the first one is in the $x$-direction and is written as

$$
\begin{array}{r}
\frac{E}{1-\nu^{2}} \frac{\partial^{2} u_{n}\left(x_{n}, y, t\right)}{\partial x_{n}^{2}}+\frac{E}{2(1-\nu)} \frac{\partial^{2} v_{n}\left(x_{n}, y, t\right)}{\partial x_{n} \partial y}+ \\
G \frac{\partial^{2} u_{n}\left(x_{n}, y, t\right)}{\partial y^{2}}=\rho \frac{\partial^{2} u_{n}\left(x_{n}, y, t\right)}{\partial t^{2}}
\end{array}
$$


and the second one is in the $y$-direction and is written as

$$
\begin{array}{r}
\frac{E}{1-\nu^{2}} \frac{\partial^{2} v_{n}\left(x_{n}, y, t\right)}{\partial x_{n}^{2}}+\frac{E}{2(1-\nu)} \frac{\partial^{2} u_{n}\left(x_{n}, y, t\right)}{\partial x_{n} \partial y}+ \\
G \frac{\partial^{2} v_{n}\left(x_{n}, y, t\right)}{\partial y^{2}}=\rho \frac{\partial^{2} v_{n}\left(x_{n}, y, t\right)}{\partial t^{2}}
\end{array}
$$

where $u_{n}\left(x_{n}, y, t\right)$ is the in-plane displacement in the $x$ direction, $v_{n}\left(x_{n}, y, t\right)$ is the in-plane displacement in the $y$ direction, $\rho$ is density, $E$ is Young's modulus, $G$ is the shear modulus, $\nu$ is Poisson's ratio, and the subscript $n$ denotes either the web $(n=w)$, left flange $(n=f l)$ or right flange $(n=f r)$. The specific orientations of the three coordinate systems to the three components of the model are depicted in Fig. 3. Note that they all share a common $y$-axis. The equation modelling the out-of-plane motion of the components in the transverse $z$-direction is the classical plate theory derived by Love and Kirchhoff. ${ }^{22}$ This equation is written as

$$
\begin{gathered}
D_{n}\left(\frac{\partial^{4} w_{n}\left(x_{n}, y, t\right)}{\partial x_{n}^{4}}+2 \frac{\partial^{4} w_{n}\left(x_{n}, y, t\right)}{\partial x_{n}^{2} \partial y^{2}}+\frac{\partial^{4} w_{n}\left(x_{n}, y, t\right)}{\partial y^{4}}\right)+ \\
\rho t_{n} \frac{\partial^{2} w_{n}\left(x_{n}, y, t\right)}{\partial t^{2}}=0
\end{gathered}
$$

where $w_{n}\left(x_{n}, y, t\right)$ is the out-of-plane displacement, $t_{n}$ is the thickness, and $D_{n}$ is the flexural rigidity and is equal to ${ }^{23}$

$$
D_{n}=\frac{E t_{n}^{3}}{12\left(1-\nu^{2}\right)}
$$

and it is noted that $t_{w}=b_{w}$ and $t_{f l}=t_{f r}=h_{f}$.

The solutions to Eqs. (1) and (2) for the in-plane motion $\operatorname{are}^{24}$

$$
u_{n}\left(x_{n}, y, t\right)=U_{n}\left(x_{n}\right) \exp \left(i k_{y} y\right) \exp (i \omega t)
$$

and

$$
v_{n}\left(x_{n}, y, t\right)=V_{n}\left(x_{n}\right) \exp \left(i k_{y} y\right) \exp (i \omega t)
$$

where

$$
\begin{aligned}
U_{w}\left(x_{w}\right)= & C_{1} \alpha \cos \left(\alpha x_{w}\right)-C_{2} \alpha \sin \left(\alpha x_{w}\right)+ \\
& C_{3} i k_{y} \sin \left(\beta x_{w}\right)+C_{4} i k_{y} \cos \left(\beta x_{w}\right) ; \\
U_{f l}\left(x_{f l}\right)= & C_{9} \alpha \cos \left(\alpha x_{f l}\right)-C_{10} \alpha \sin \left(\alpha x_{f l}\right)+ \\
& C_{11} i k_{y} \sin \left(\beta x_{f l}\right)+C_{12} i k_{y} \cos \left(\beta x_{f l}\right) ; \\
U_{f r}\left(x_{f r}\right)= & C_{17} \alpha \cos \left(\alpha x_{f r}\right)-C_{18} \alpha \sin \left(\alpha x_{f r}\right)+ \\
& C_{19} i k_{y} \sin \left(\beta x_{f r}\right)+C_{20} i k_{y} \cos \left(\beta x_{f r}\right) ; \\
V_{w}\left(x_{w}\right)= & C_{1} i k_{y} \sin \left(\alpha x_{w}\right)+C_{2} i k_{y} \cos \left(\alpha x_{w}\right)- \\
& C_{3} \beta \cos \left(\beta x_{w}\right)+C_{4} \beta \sin \left(\beta x_{w}\right) ; \\
V_{f l}\left(x_{f l}\right)= & C_{9} i k_{y} \sin \left(\alpha x_{f l}\right)+C_{10} i k_{y} \cos \left(\alpha x_{f l}\right)- \\
& C_{11} \beta \cos \left(\beta x_{f l}\right)+C_{12} \beta \sin \left(\beta x_{f l}\right) ;
\end{aligned}
$$

and

$$
\begin{aligned}
V_{f r}\left(x_{f r}\right)= & C_{17} i k_{y} \sin \left(\alpha x_{f r}\right)+C_{18} i k_{y} \cos \left(\alpha x_{f r}\right)- \\
& C_{19} \beta \cos \left(\beta x_{f r}\right)+C_{20} \beta \sin \left(\beta x_{f r}\right) .
\end{aligned}
$$

In Eqs. (7)-(12), $\alpha$ and $\beta$ are modified wavenumbers and are equal to ${ }^{24}$

$$
\alpha=\sqrt{k_{p}^{2}-k_{y}^{2}}
$$

and

$$
\beta=\sqrt{k_{s}^{2}-k_{y}^{2}}
$$

where $k_{p}$ is the plate wavenumber expressed as

$$
k_{p}=\frac{\omega}{c_{p}}=\frac{\omega}{\sqrt{\frac{E}{\left(1-\nu^{2}\right) \rho}}}
$$

and $k_{s}$ is the shear wavenumber expressed as

$$
k_{s}=\frac{\omega}{c_{s}}=\frac{\omega}{\sqrt{\frac{G}{\rho}}}
$$

and the constants $C_{i}$ in Eqs. (7)-(12) are in-plane wave propagation coefficients and are determined from the boundary conditions of the system.

The solutions to Eq. (3) for the out-of-plane motion are ${ }^{24}$

$$
w_{n}\left(x_{n}, y, t\right)=W_{n}\left(x_{n}\right) \exp \left(i k_{y} y\right) \exp (i \omega t)
$$

where

$$
\begin{aligned}
W_{w}\left(x_{w}\right)= & C_{5} \sin \left(\xi_{1} x_{w}\right)+C_{6} \cos \left(\xi_{1} x_{w}\right)+ \\
& C_{7} \sinh \left(\xi_{2} x_{w}\right)+C_{8} \cosh \left(\xi_{2} x_{w}\right) ; \\
W_{f l}\left(x_{f l}\right)= & C_{13} \sin \left(\xi_{3} x_{f l}\right)+C_{14} \cos \left(\xi_{3} x_{f l}\right)+ \\
& C_{15} \sinh \left(\xi_{4} x_{f l}\right)+C_{16} \cosh \left(\xi_{4} x_{f l}\right) ;
\end{aligned}
$$

and

$$
\begin{aligned}
W_{f r}\left(x_{f r}\right)= & C_{21} \sin \left(\xi_{3} x_{f r}\right)+C_{22} \cos \left(\xi_{3} x_{f r}\right)+ \\
& C_{23} \sinh \left(\xi_{4} x_{f r}\right)+C_{24} \cosh \left(\xi_{4} x_{f r}\right) .
\end{aligned}
$$

In Eqs. (18)-(20), the $\xi_{i}$ 's are plate wavenumbers and are equal to

$$
\begin{aligned}
& \xi_{1}=\left(\sqrt{\frac{\rho b_{w} \omega^{2}}{D_{w}}}-k_{y}^{2}\right)^{\frac{1}{2}} ; \\
& \xi_{2}=\left(\sqrt{\frac{\rho b_{w} \omega^{2}}{D_{w}}}+k_{y}^{2}\right)^{\frac{1}{2}} ; \\
& \xi_{3}=\left(\sqrt{\frac{\rho h_{f l} \omega^{2}}{D_{f l}}}-k_{y}^{2}\right)^{\frac{1}{2}}=\left(\sqrt{\frac{\rho h_{f r} \omega^{2}}{D_{f r}}}-k_{y}^{2}\right)^{\frac{1}{2}} ;
\end{aligned}
$$

and

$$
\xi_{4}=\left(\sqrt{\frac{\rho h_{f l} \omega^{2}}{D_{f l}}}+k_{y}^{2}\right)^{\frac{1}{2}}=\left(\sqrt{\frac{\rho h_{f r} \omega^{2}}{D_{f r}}}+k_{y}^{2}\right)^{\frac{1}{2}} .
$$

Note that the solution to the plate equations assume $\rho t_{n} \omega^{2} / D_{n} \neq k_{y}^{2}$. When the quartic equation $\rho t_{n} \omega^{2} / D_{n}=k_{y}^{2}$ is satisfied, a different solution to Eq. (3) will result. However, in the unlikely event this occurs, a very small value can be added or subtracted to one of the wavenumbers to make Eqs. (18)-(20) the admissible solution. The constants $C_{i}$ in Eqs. (18)-(20) are out-of-plane wave propagation coefficients and are also determined from the boundary conditions of the system. 
The various forces of the structure are now mathematically defined. These will be used in the force and moment balance equations to solve for the $C_{i}$ 's. The normal in-plane forces are determined using ${ }^{23}$

$$
N_{x x}^{(n)}\left(x_{n}\right)=\frac{E t_{n}}{1-\nu^{2}}\left[\frac{d U_{n}\left(x_{n}\right)}{d x_{n}}+i k_{y} \nu V_{n}\left(x_{n}\right)\right]
$$

and the shear in-plane forces are ${ }^{23}$

$$
N_{x y}^{(n)}\left(x_{n}\right)=\frac{E t_{n}}{2(1+\nu)}\left[i k_{y} U_{n}\left(x_{n}\right)+\frac{d V_{n}\left(x_{n}\right)}{d x_{n}}\right] .
$$

The shear out-of-plane forces are calculated using ${ }^{25}$

$$
V_{x}^{(n)}\left(x_{n}\right)=-D_{n}\left[\frac{d^{3} W_{n}\left(x_{n}\right)}{d x_{n}^{3}}-(2-\nu) k_{y}^{2} \frac{d W_{n}\left(x_{n}\right)}{d x_{n}}\right] ;
$$

and the moments are determined with ${ }^{25}$

$$
M_{x x}^{(n)}\left(x_{n}\right)=-D_{n}\left[\frac{d^{2} W_{n}\left(x_{n}\right)}{d x_{n}^{2}}-\nu k_{y}^{2} W_{n}\left(x_{n}\right)\right] .
$$

There are 24 equations that constitute the boundary conditions of this system. The boundary conditions at the top of the web $\left(x_{w}=h_{w}\right)$ are

$$
\begin{aligned}
& N_{x x}^{(w)}\left(h_{w}\right)=-b_{w} P_{0} \\
& N_{x y}^{(w)}\left(h_{w}\right)=-b_{w} F_{0} \\
& V_{x}^{(w)}\left(h_{w}\right)=-b_{w} Q_{0}
\end{aligned}
$$

and

$$
M_{x x}^{(w)}\left(h_{w}\right)=0
$$

where $P_{0}$ is the normal external pressure acting in the $x$ direction of the web, $F_{0}$ is the axial external pressure acting in the $y$-direction of the web, and $Q_{0}$ is the transverse external pressure acting in the $z$-direction of the web. Implicit in these pressure loads is the multiplication of exponential functions in $y$-direction wavenumber and frequency. In general, the most important loading quantity is the normal pressure. Note that these forcing functions act on the top of the web, as this model allows the beam to be loaded at a location other than the neutral axis of the beam, and this corresponds more closely to the actual physical problem than loading the beam on its neutral axis. A moment load is also possible but was not investigated in this work because it is not of interest to the particular problem being solved. The four boundary conditions at the free end of the left flange $\left(x_{f l}=a\right)$ are

$$
N_{x x}^{(f l)}(a)=N_{x y}^{(f l)}(a)=V_{x}^{(f l)}(a)=M_{x x}^{(f l)}(a)=0 ;
$$

where it is noted that $a<0$. Similarly, the four boundary conditions at the free end of the right flange $\left(x_{f l}=b\right)$ are

$$
N_{x x}^{(f r)}(b)=N_{x y}^{(f r)}(b)=V_{x}^{(f r)}(b)=M_{x x}^{(f r)}(b)=0 .
$$

There are three force balances at the intersection of the web and flange $\left(x_{w}=x_{f l}=x_{f r}=0\right)$ and these are written as

$$
\begin{aligned}
& N_{x x}^{(w)}(0)-V_{x}^{(f l)}(0)+V_{x}^{(f r)}(0)=0 ; \\
& V_{x}^{(w)}(0)+N_{x x}^{(f l)}(0)-N_{x x}^{(f r)}(0)=0 ;
\end{aligned}
$$

and

$$
N_{x y}^{(w)}(0)-N_{x y}^{(f l)}(0)+N_{x y}^{(f r)}(0)=0 ;
$$

and there is a moment balance at this location, written as

$$
M_{x x}^{(w)}(0)-M_{x x}^{(f l)}(0)+M_{x x}^{(f r)}(0)=0 .
$$

Note that these force and moment equations converge on a line along the $y$-axis of the structure where the web, left flange, and right flange intersect. In an actual beam, these forces are distributed over a small volume of the material at this location. There are eight continuity equations at the intersection and these are written as

$$
\begin{gathered}
u_{w}(0)=w_{f l}(0)=w_{f r}(0) ; \\
w_{w}(0)=-u_{f l}(0)=-u_{f r}(0) ; \\
v_{w}(0)=v_{f l}(0)=v_{f r}(0) ;
\end{gathered}
$$

and

$$
\frac{d w_{w}(0)}{d x_{w}}=\frac{d w_{f l}(0)}{d x_{f l}}=\frac{d w_{f r}(0)}{d x_{f r}} .
$$

Inserting Eqs. (7)-(12) and (18)-(20) into Eqs. (29)-(42) produces a 24 by 24 algebraic matrix equation given by

$$
[\mathbf{A}]\{\mathbf{x}\}=\{\mathbf{b}\}
$$

where the entries of $[\mathbf{A}]$ are in Appendix A as Eqs. (A.1) to (A.104), the vector $\{\mathbf{x}\}$ is

$$
\{\mathbf{x}\}=\left\{\begin{array}{lllll}
C_{1} & C_{2} & \ldots & C_{23} & C_{24}
\end{array}\right\}^{T} ;
$$

and the $\{\mathbf{b}\}$ vector is

$$
\{\mathbf{b}\}=\left\{\begin{array}{llllllll}
-b_{w} P_{0} & -b_{w} F_{0} & -b_{w} Q_{0} & 0 & 0 & \ldots & 0 & 0
\end{array}\right\}^{T} .
$$

The solution to the wave propagation coefficients $C_{i}$ in Eq. (43) is found using

$$
\{\mathbf{x}\}=[\mathbf{A}]^{-1}\{\mathbf{b}\}
$$

and once these are known, they can be inserted into Eqs. (7), (10), and (18) and the displacement response of the web for external loading in three dimensions can be calculated. Additionally, the displacement of the flange can also be calculated, but it is typically not a quantity of interest.

To integrate this beam model into a reinforced structural model, the dynamic stiffness components of the beam are typically calculated and used. For a symmetric T-beam, there are four unique and nonzero terms. The first term is the dynamic stiffness of the normal displacement to normal pressure and is written as

$$
K_{z z}=\frac{-b_{w} P_{0}}{U_{w}\left(h_{w}\right)}
$$

the second term is the dynamic stiffness of normal displacement to axial pressure (and equal to axial displacement to normal pressure), and is written as

$$
K_{z y}=K_{y z}=\frac{-b_{w} F_{0}}{U_{w}\left(h_{w}\right)}=\frac{-b_{w} P_{0}}{V_{w}\left(h_{w}\right)} ;
$$


the third term is the dynamic stiffness of axial displacement to axial pressure, and is written as

$$
K_{y y}=\frac{-b_{w} F_{0}}{V_{w}\left(h_{w}\right)}
$$

and the fourth term is the dynamic stiffness of transverse displacement to transverse pressure, and is written as

$$
K_{x x}=\frac{-b_{w} Q_{0}}{W_{w}\left(h_{w}\right)}
$$

where the units of Eqs. (47)-(50) are stiffness per unit length. Finally, it is noted that if a second flange is present on the top of the beam, i.e. an I- or an H-beam design, this dynamic contribution can be added to the model in the same method as the bottom flange equations.

\section{REDUCED MODEL}

The complexity of the model derived in Section 2 can be reduced considerably if the beam is symmetric and only in-plane loading is of interest. The flexural wave motion component of the web is zero and the flange contributions can be rewritten as forces acting on the web equations of motion at the intersection of the web and flange. This will produce a four by four system of equations written as

$$
\left[\begin{array}{cccc}
a_{1,1} & a_{1,2} & a_{1,3} & a_{1,4} \\
a_{2,1} & a_{2,2} & a_{2,3} & a_{2,4} \\
b_{3,1} & a_{13,2} & a_{13,3} & b_{3,4} \\
a_{15,1} & b_{4,2} & b_{4,3} & a_{15,4}
\end{array}\right]\left\{\begin{array}{l}
C_{1} \\
C_{2} \\
C_{3} \\
C_{4}
\end{array}\right\}=\left\{\begin{array}{c}
-b_{w} P_{0} \\
-b_{w} F_{0} \\
0 \\
0
\end{array}\right\}
$$

where

$$
\begin{aligned}
b_{3,1}= & 2 D_{f r} \alpha \xi_{3}^{2} \xi_{4}^{2}\left(\xi_{3}^{2}+\xi_{4}^{2}\right)\left[\xi_{3} \sin \left(\xi_{3} b\right) \cosh \left(\xi_{4} b\right)+\right. \\
& \left.\xi_{4} \cos \left(\xi_{3} b\right) \sinh \left(\xi_{4} b\right)\right] / \Delta_{z} ; \\
b_{3,4}= & 2 D_{f r} i k_{y} \xi_{3}^{2} \xi_{4}^{2}\left(\xi_{3}^{2}+\xi_{4}^{2}\right)\left[\xi_{3} \sin \left(\xi_{3} b\right) \cosh \left(\xi_{4} b\right)+\right. \\
& \left.\xi_{4} \cos \left(\xi_{3} b\right) \sinh \left(\xi_{4} b\right)\right] / \Delta_{z} ; \\
b_{4,2}= & \frac{E}{1+\nu} h_{f} \alpha i k_{y}\left(\beta^{2}+k_{y}^{2}\right) \sin (\alpha b) \sin (\beta b) / \Delta_{x} ; \\
b_{4,3}= & \frac{-E}{1+\nu} h_{f} \alpha \beta\left(\beta^{2}+k_{y}^{2}\right) \sin (\alpha b) \sin (\beta b) / \Delta_{x} ; \\
\Delta_{z}= & \xi_{3}^{4}+\xi_{4}^{4}+\left(\xi_{3}^{3} \xi_{4}-\xi_{3} \xi_{4}^{3}\right) \sin \left(\xi_{3} b\right) \sinh \left(\xi_{4} b\right)+ \\
& 2 \xi_{3}^{2} \xi_{4}^{2} \cos \left(\xi_{3} b\right) \cosh \left(\xi_{4} b\right) ;
\end{aligned}
$$

and

$$
\Delta_{x}=k_{y}^{2} \cos (\alpha b) \sin (\beta b)+\alpha \beta \sin (\alpha b) \cos (\beta b) .
$$

Equations (7) and (10) are used to calculate the in-plane normal and in-plane axial displacement of the web, respectively, and the out-of-plane transverse displacement is not calculated (or is set to zero) as the symmetric model cannot admit torsional motion. The reduced model will produce identical normal and axial results compared to the full model derived in Section 2 for symmetric beams. Note that if the flange is absent, the beam can still be modelled by setting the matrix elements $b_{3,1}$, $b_{3,4}, b_{4,2}$, and $b_{4,3}$ to zero.

\section{EXAMPLE PROBLEM}

The model is now analysed using an example problem, where the beam has material and geometric properties that are consistent with an application to underwater structures. The Tbeam has the following physical dimensions: height of the web $h_{w}=0.2032 \mathrm{~m}$ (8.0 in), width of the web $b_{w}=0.0127 \mathrm{~m}$ (0.5 in), height of the flange $h_{f}=0.0064 \mathrm{~m}(0.25 \mathrm{in})$, and width of the flange $b_{f}=0.127 \mathrm{~m}$ (5.0 in), which results in the left flange free end at $a=-b_{f} / 2=-0.0635 \mathrm{~m}$ and the right flange free end at $b=b_{f} / 2=0.0635 \mathrm{~m}$. The beam is made of steel which has the following mechanical properties: Young's modulus $E=200 \times 10^{9} \mathrm{~N} \mathrm{~m}^{-2}$, shear modulus $G=76.92 \times 10^{9} \mathrm{~N} \mathrm{~m}^{-2}$, Poisson's ratio $\nu=0.30$, and density $\rho=7800 \mathrm{~kg} \mathrm{~m}^{-3}$. The beam is independently loaded on its top surface with three separate loads that correspond to normal (web in-plane), axial (web in-plane), and transverse (web out-of-plane) pressure. Although any location of the beam can be chosen for the displacement output, the top of the web is investigated here because this location is pertinent to the analysis of reinforced structures. This allows the dynamic stiffness of the beam to be calculated and subsequently used in the analysis of beams attached to plates or elastic bodies. Thus, the output of the model is the normal, axial, and transverse beam displacement at the top of the web. It is noted, however, that by far the most important model output is the normal displacement divided by normal pressure, as this corresponds to the main design objective of most beams. Plots of the other outputs are included for completeness. Performance metrics in the form of displacement comparisons that are included later in this section are calculated for normal displacement divided by normal pressure.

There are nine combinations of displacements divided by pressures. Figure 4 is a plot of normal displacement divided by normal pressure versus axial $\left(k_{y}\right)$ wavenumber at $5 \mathrm{kHz}$. The solid line is the model developed in Section 2, the dashed line is the Bickford beam model and the $\times$ markers are results from finite element analysis. The Bickford beam results were found solving the differential equations that Bickford derived to correspond to an infinite length beam and calculating the normal displacement response and a condensed set of these equations are presented in Appendix B. ${ }^{3}$ The Bickford beam results (in Fig. 4) are for a beam geometry that contains both the web and the flange. The finite element model results were produced using COMSOL 5.2a, using a model that consisted of 1850 quadratic serendipity hexahedral elements and a total of 42,234 degrees of freedom. As these elements are continuum elements, the response of the finite element model is fully elastic. Figure 5 is a plot of axial displacement divided by normal pressure versus axial wavenumber. In this plot, the axial displacement is zero at zero wavenumber because normal pressure (when $k_{y}=0$ ) is spatially invariant and this can only result in a displacement field that is normal translation, i.e. the axial translation is zero. Figure 6 is a plot of axial displacement divided by axial pressure versus axial wavenumber. The plot of normal displacement divided by axial pressure is not included as it is identical to Fig. 5. Figure 7 is a plot of transverse displacement divided by transverse pressure versus axial wavenumber. In this plot, the analytical model results are 


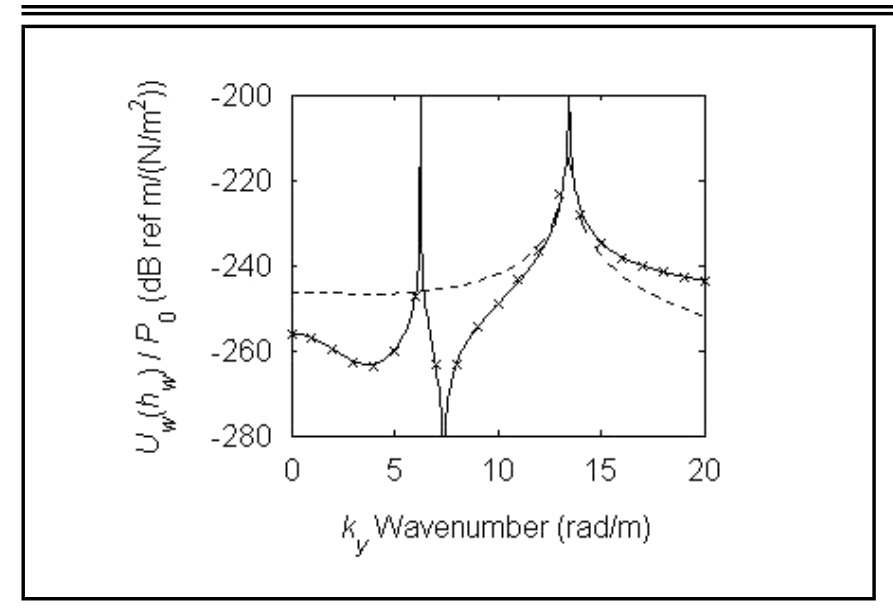

Figure 4. Normal displacement divided by normal pressure versus wavenumber at $5 \mathrm{kHz}$. The solid line is the new model, the dashed line is the Bickford beam model, and the $\times$ markers are finite element results.

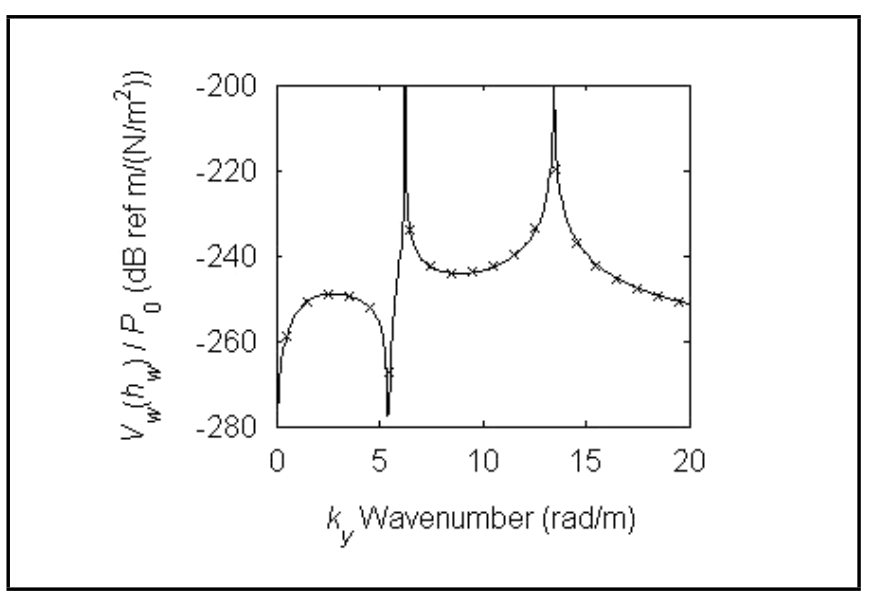

Figure 5. Axial displacement divided by normal pressure versus wavenumber at $5 \mathrm{kHz}$. The solid line is the new model and the $\times$ markers are finite element results.

stiffer than the finite element results, which is expected, because most of the energy is flexural wave motion and the classical plate equation results in a model that is somewhat too stiff at these frequencies. In Figs. 5, 6, and 7, the solid line is the new model and the $\times$ markers are results from finite element analysis. The model results of normal displacement divided by transverse pressure, axial displacement divided by transverse pressure, transverse displacement divided by normal pressure, and transverse displacement divided by axial pressure are not shown, as they are all zero or extremely close to zero. In Figs. 4-7, the peak displacement values occur when an excitation wavenumber and frequency match a resonant condition in the beam and the result is an unbounded response. Mathematically, this corresponds to $\operatorname{det}[\mathbf{A}]=0$ in Eq. (43). These peaks do not always occur at the same wavenumbers in the different plots because the in-plane resonant waves are not coupled to the out-of-plane resonant waves in this model.

The response of the system is also studied for zero axial wavenumber response. Figure 8 is a plot of normal displacement divided by normal pressure versus frequency at zero wavenumber. The solid line is the new model, the dashed line is the Bickford beam model, and the $\times$ markers are results from finite element analysis. Although the Bickford beam model can predict the flexural wave resonant response, it does

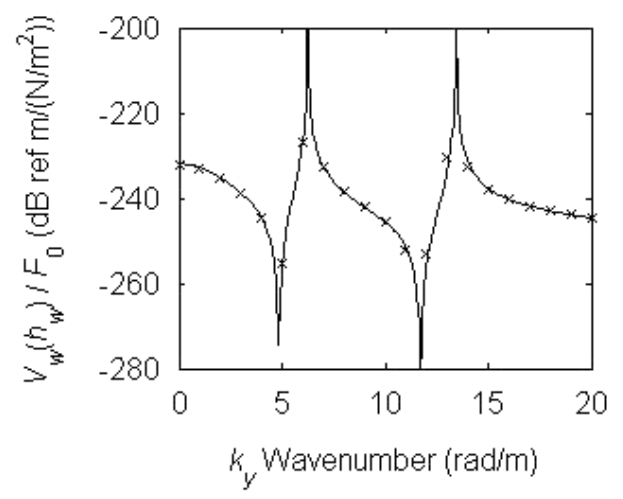

Figure 6. Axial displacement divided by axial pressure versus wavenumber at $5 \mathrm{kHz}$. The solid line is the new model and the $\times$ markers are finite element results.

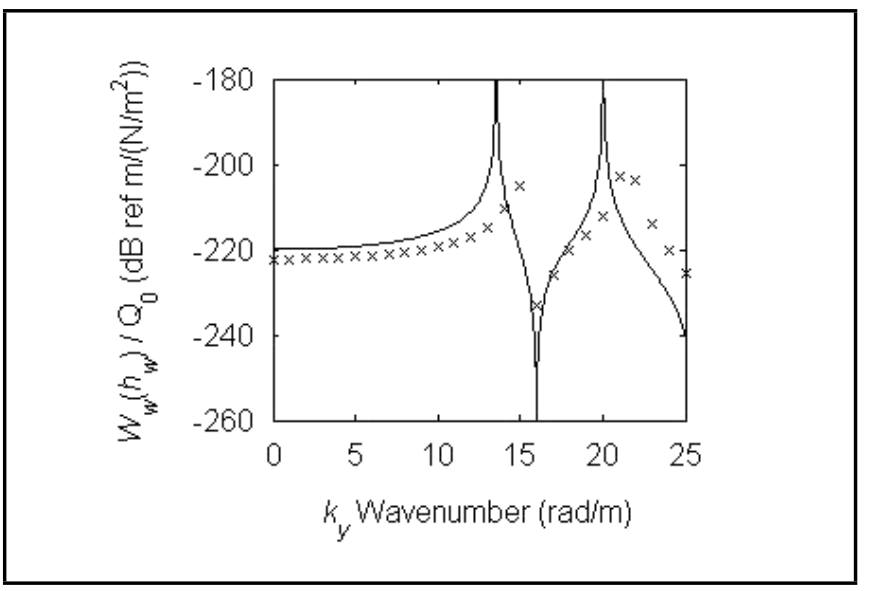

Figure 7. Transverse displacement divided by transverse pressure versus wavenumber at $5 \mathrm{kHz}$. The solid line is the new model and the $\times$ markers are finite element results.

not have the degrees of freedom necessary to incorporate the higher order wave dynamics that are present across the web of the beam that are exhibited in the new model and finite element response, present in Fig. 8 at frequencies of approximately 1.5 and $8.2 \mathrm{kHz}$. Furthermore, the Bickford beam model does not have the proper phase angle response at higher frequencies, and this effect is clearly visible above $5.7 \mathrm{kHz}$. The divergence of the analytical model and the finite element model at approximately $8 \mathrm{kHz}$ is due to increased out-of-plane dynamics interacting with the in-plane dynamics; the out-of-plane model is (slightly) too stiff at these frequencies. Figure 9 is a plot of axial displacement divided by axial pressure versus frequency and Fig. 10 is a plot of transverse displacement divided by transverse pressure versus frequency. In Figs. 9 and 10 , the solid line is the new model and the $\times$ markers are results from finite element analysis. As the structure is undergoing extensive out-of-plane motion in the loading associated with Fig. 10, the analytical model begins to diverge from the finite element results around $6 \mathrm{kHz}$. The resonances around 1.5 and $8.2 \mathrm{kHz}$ in Fig. 8 are higher order branch modes that have cut-off frequencies similar to higher order plate modes. This is illustrated in Fig. 11, which is a plot of the flexural wave location of the beam in the wavenumber-frequency plane, where the solid line is the new beam model, the dashed line is the 


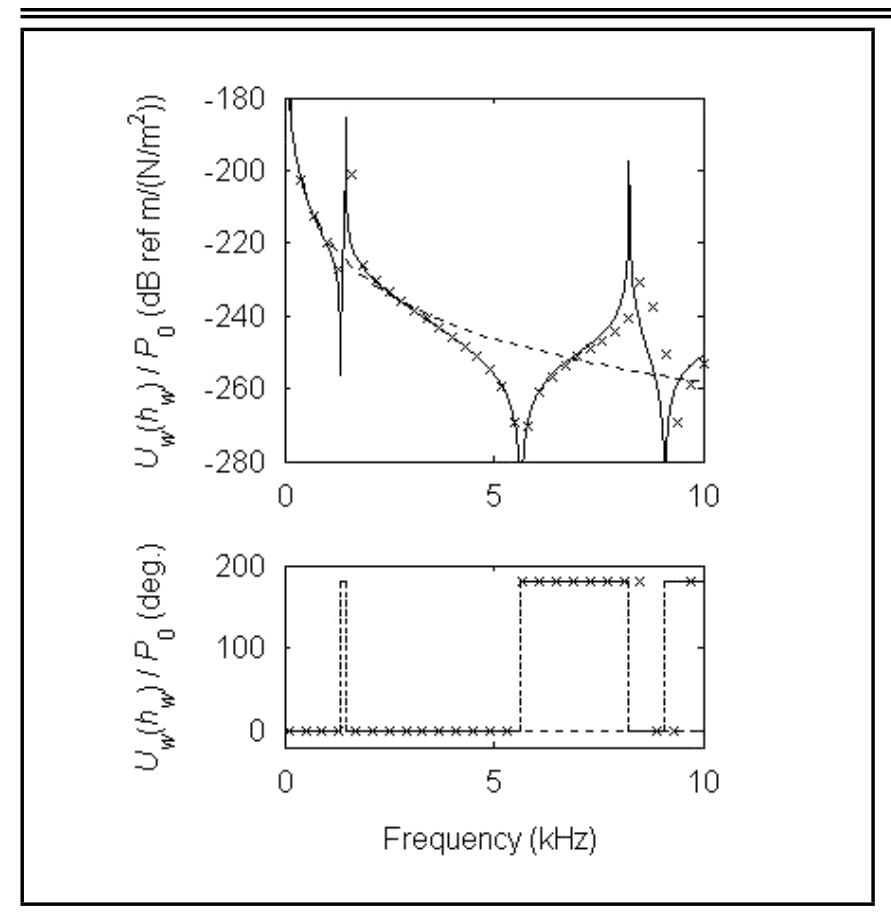

Figure 8. Normal displacement divided by normal pressure magnitude (top) and phase angle (bottom) versus frequency at zero wavenumber. The solid line is the new beam model, the dashed line is the Bickford beam model, and the $\times$ markers are the finite element results.

Bickford beam model, and the $\times$ markers are finite element results. These cut-off frequencies can be predicted at $k_{y}=0$ by setting the determinant of the matrix in Eq. (51), denoted B, equal to zero, given by the equation

$$
\operatorname{det}\left[\mathbf{B}\left(k_{y}=0\right)\right]=0 ;
$$

which results in

$$
K_{f} c_{p} \cos \left(\frac{\omega h_{w}}{c_{p}}\right)+\left(\frac{E}{1-\nu^{2}}\right) \omega \sin \left(\frac{\omega h_{w}}{c_{p}}\right)=0
$$

and

$b_{w} \cos \left(\frac{\omega b}{c_{s}}\right) \sin \left(\frac{\omega h_{w}}{c_{s}}\right)+2 h_{f} \sin \left(\frac{\omega b}{c_{s}}\right) \cos \left(\frac{\omega h_{w}}{c_{s}}\right)=0 ;$

where

$$
K_{f}=\frac{2 D_{f r} \xi^{3}[\cos (\xi b) \sinh (\xi b)+\cosh (\xi b) \sin (\xi b)]}{\cos (\xi b) \cosh (\xi b)+1}
$$

and

$$
\xi=\left(\frac{\rho h_{f} \omega^{2}}{D_{f r}}\right)^{\frac{1}{4}}
$$

A higher order wave at a corresponding cut-off frequency for normal motion occurs when the function in Eq. (59) is equal to zero, and for this beam, these frequencies are at 1.46 and $8.24 \mathrm{kHz}$ in the frequency range from 0 to $10 \mathrm{kHz}$. Similarly, a higher order wave at a corresponding cut-off frequency for axial motion occurs when the function in Eq. (60) is equal to zero, and for this beam, this frequency is at $5.88 \mathrm{kHz}$ in the frequency range from 0 to $10 \mathrm{kHz}$.

The new model has compared very favourably to the solution generated using finite element analysis for the example problem. The smallest deviation between the new model and
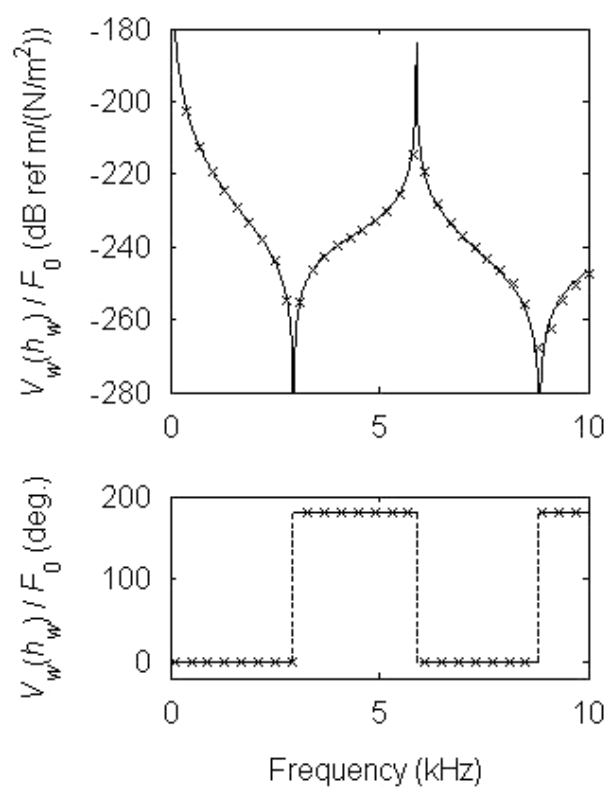

Figure 9. Axial displacement divided by axial pressure magnitude (top) and phase angle (bottom) versus frequency at zero wavenumber. The solid line is the new beam model and the $\times$ markers are the finite element results.

the finite element results is axial displacement divided by axial force, shown in Fig. 9. This is due to the wave propagation of the flange and web which is restricted to in-plane motion and this is very accurately modelled by the two-dimensional equations of elasticity in both beam sections in the model. The next smallest deviation is the normal displacement divided by normal force, shown in Fig. 8. For this model output, the motion in the web is in-plane and the motion in the flange is predominantly out-of-plane. The out-of-plane equation of motion has lower frequency limits on its accuracy than the inplane equations of motion and this effect is observed around $8.4 \mathrm{kHz}$, where the new model is beginning to become stiffer than the finite element model, specifically due to the LoveKirchhoff plate model that is used in this analysis. The transverse displacement divided by transverse pressure model compares favourably to the finite element results to about $6 \mathrm{kHz}$, and then some divergence is noted. This displacement is typically much less important in beam design than the normal displacement, however, if this is an important quantity, then a higher order model, such as a Mindlin plate or a Reddy plate, can be used instead of a Love-Kirchhoff plate. Inclusion of these thick plate models will increase complexity of the new model.

The results are now examined using a comparison of the wavenumber locations of the flexural resonant peaks and the responses of displacement at zero wavenumber at ten frequencies. Table 1 is a comparison of the flexural wavenumber differences between the new beam model and the Bickford beam model, compared to the finite element model calculated using the equation

$$
P_{1}(f)=\frac{k_{F E M}-k_{M}}{k_{F E M}} \cdot 100 \% ;
$$

where $P_{1}(f)$ is the normalized difference in flexural wave locations expressed in percent, $k_{F E M}$ is the flexural wavenum- 


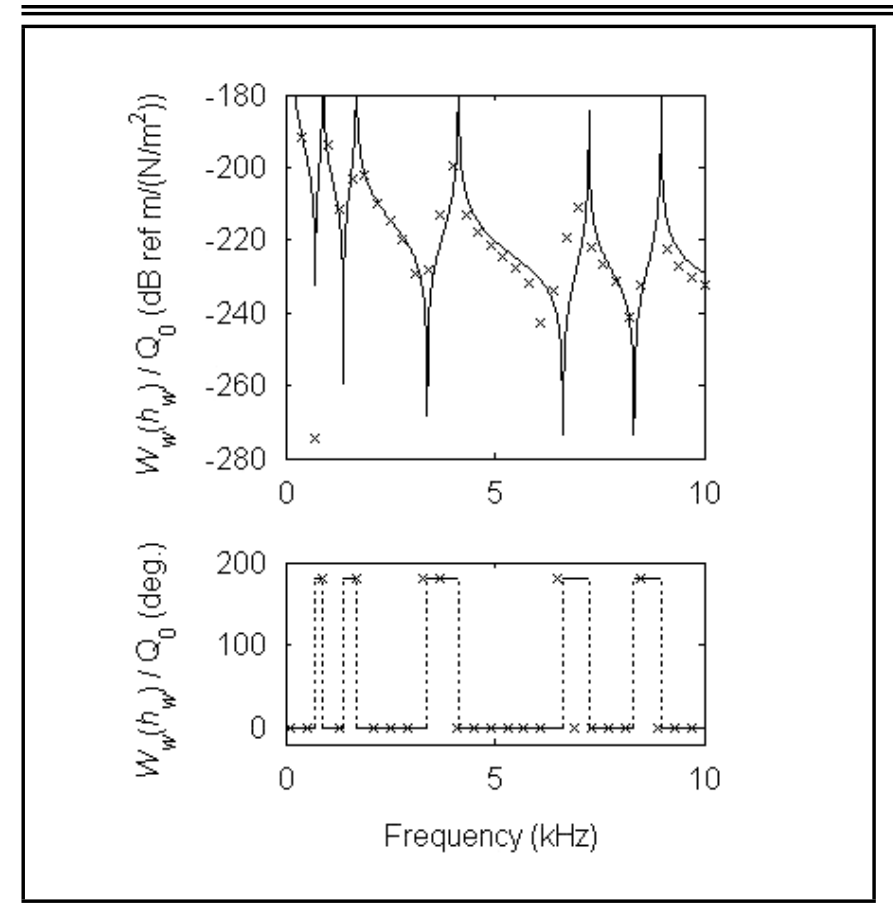

Figure 10. Transverse displacement divided by transverse pressure magnitude (top) and phase angle (bottom) versus frequency at zero wavenumber. The solid line is the new beam model and the $\times$ markers are the finite element results.

ber of the finite element model, and $k_{M}$ is the flexural wavenumber of the analytical models. Using this equation produces positive values from smaller (stiffer system response) flexural wavenumbers and negative values from larger (softer system response) flexural wavenumbers. Table 2 is a comparison of web normal displacement divided by normal pressure at zero wavenumber between the new beam model and the Bickford beam model, compared to the finite element model calculated using the equation

$$
P_{2}(f)=\frac{\left[U_{w}\left(h_{w}\right) / P_{0}\right]_{F E M}-\left[U_{w}\left(h_{w}\right) / P_{0}\right]_{M}}{\left[U_{w}\left(h_{w}\right) / P_{0}\right]_{F E M}} \cdot 100 \%
$$

where $P_{2}(f)$ is the normalized difference of zero wavenumber amplitude expressed in percent, $\left[U_{w}\left(h_{w}\right) / P_{0}\right]_{F E M}$ is the amplitude of the finite element model and $\left[U_{w}\left(h_{w}\right) / P_{0}\right]_{M}$ is the amplitude of the analytical models. Using this equation produces positive values from smaller (softer system response) amplitudes and negative values from larger (stiffer system response) amplitudes.

\section{CONCLUSIONS}

An analytical beam model for a T-shaped beam was derived and compared to a Bickford beam model and a finite element model. This new model was constructed with two-dimensional elastic equations for the in-plane motion and classical plate equations for the out-of-plane motion. This allows for a relatively elastic response of the entire system. It was shown that this model increases the frequency range analysis by a factor of eight, compared to the Bickford beam model for the example presented here. This upper-frequency range could be increased by replacing the classical plate equations by higher order plate equations. The application of this model to a reinforced structure is discussed. A simplified version of the model for a sym-

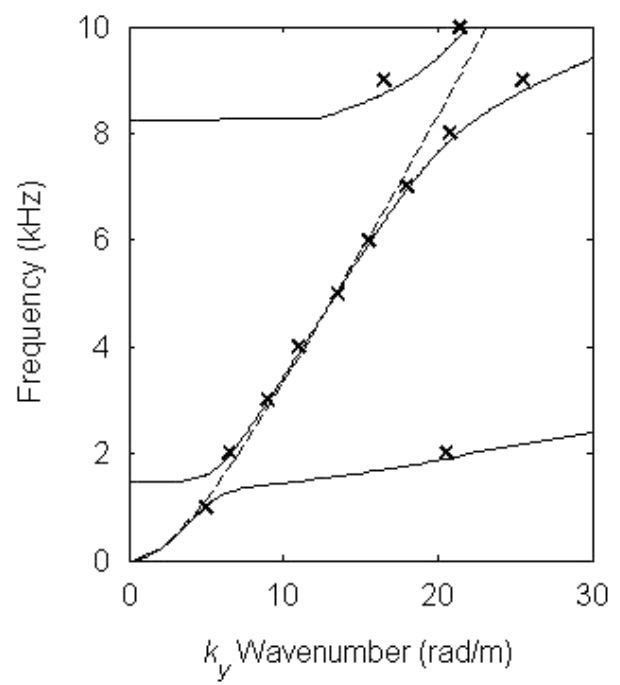

Figure 11. Location of the flexural wave in the wavenumber-frequency plane. The solid line is the new beam model, the dashed line is the Bickford beam model, and the $\times$ markers are the finite element results.

metric structure with normal and axial web in-plane loading is presented. It is recommended that future work apply this new beam model to the problem of analysis of a reinforced plate.

\section{ACKNOWLEDGEMENTS}

This work was funded by the Naval Undersea Warfare Centre's (NUWC) In-House Laboratory Independent Research (ILIR) Program.

\section{REFERENCES}

1 Euler, L. The elastic curve, methods for finding curved lines enjoying properties of maximum or minimum, Michaelem Bousquet \& Socios., Lausanne, Switzerland, (1744).

2 Timoshenko, S. P. On the transverse vibrations of bars of uniform cross-section, The London, Edinburgh, and Dublin Philosophical Magazine and Journal of Science, 43 (253), 125-131, (1922). https://dx.doi.org/10.1080/14786442208633855

3 Bickford, W. B. A consistent higher order beam theory, 11th Southeastern Conference on Theoretical and Applied Mechanics, Huntsville, AL, 11, 137-150, (1982).

4 Karama, M., Afaq, K. S., and Mistou, S. Mechanical behaviour of laminated composite beam by new multi-layered laminated composite structures model with transverse shear stress continuity, International Journal of Solids and Structures, 40 (6), 1525-1546, (2003). https://dx.doi.org/10.1016/S0020-7683(02)00647-9

5 Mindlin, R. D. Influence of rotary inertia and shear on flexural motions of isotropic elastic plates, ASME Journal of Applied Mechanics, 18 (1), 31-38, (1951).

6 Reddy, J. N. and Phan, N. D. Stability and vibration of isotropic, orthotropic and laminated plates accord- 
Table 1. Percent difference in flexural wave locations at various frequencies.

\begin{tabular}{|c|c|c|}
\hline Frequency $(\mathrm{kHz})$ & New Model & Bickford Model \\
\hline 1 & 0.6 & 6.7 \\
2 & -1.6 & 9.0 \\
3 & -0.6 & -3.1 \\
4 & -2.2 & -3.1 \\
5 & 0.3 & 0.3 \\
6 & -1.2 & 0.6 \\
7 & -1.1 & 3.4 \\
8 & -2.7 & 6.9 \\
9 & -9.5 & -29.0 \\
10 & -1.9 & -7.7 \\
\hline
\end{tabular}

Table 2. Percent difference of zero wavenumber displacement at various frequencies.

\begin{tabular}{|c|c|c|}
\hline Frequency $(\mathrm{kHz})$ & New Model & Bickford Model \\
\hline 1 & 5.7 & -16.3 \\
2 & 6.0 & 25.6 \\
3 & 0.1 & -4.3 \\
4 & -0.7 & 46.2 \\
5 & -1.1 & -210.9 \\
6 & -6.2 & 583.9 \\
7 & -11.2 & 191.7 \\
8 & -95.0 & 128.2 \\
9 & 109.5 & 132.2 \\
10 & -31.0 & 156.6 \\
\hline
\end{tabular}

ing to a higher-order shear deformation theory, Journal of Sound and Vibration, 98 (2), 157-170, (1985). https://dx.doi.org/10.1016/0022-460X(85)90383-9

7 Park, D. H., Hong, S. Y., Kil, H. G., and Jeon, J. J. Power flow models and analysis of in-plane waves in finite coupled thin plates, Journal of Sound and Vibration, 244 (4), 651668, (2001). https://dx.doi.org/10.1006/jsvi.2000.3517

${ }^{8}$ Kessissoglou, N. J. Power transmission in L-shaped plates including flexural and in-plane vibration, The Journal of the Acoustical Society of America, 115 (3), 1157-1169, (2004). https://dx.doi.org/10.1121/1.1635415

${ }^{9}$ Du, J., Li, W. L., Liu, Z., Yang, T., and Jin, G. Free vibration of two elastically coupled rectangular plates with uniform elastic boundary restraints, Journal of Sound and Vibration, 330 (4), 788-804, (2011). https://dx.doi.org/10.1016/j.jsv.2010.08.044

${ }^{10}$ Chen, Y., Jin, G., Zhu, M., Liu, Z., Du, J., and Li, W. L. Vibration behaviours of a box-type structure built up by plates and energy transmission through the structure, Journal of Sound and Vibration, 331 (4), 849-867, (2012). https://dx.doi.org/10.1016/j.jsv.2011.10.002

11 Wang, X. Dynamic behaviour of finite coupled Mindlin plates with a blocking mass, ASME Journal of Vibration and Acoustics, 138 (6), 061008, (2016). https://dx.doi.org/10.1115/1.4034251

12 Abdul-Ahad, R. B. and Aziz. O. Q. Flexural strength of reinforced concrete T-Beams with steel fibers, $\mathrm{Ce}$ ment and Concrete Composites, 21 (4), 263-268, (1999). https://dx.doi.org/10.1016/S0958-9465(99)00009-8
${ }_{13}$ Rahal, K. Combined torsion and bending in reinforced and prestressed concrete beams using simplified method for combined stress-resultants, Aci Structural Journal, 104 (4), 402-411, (2007). https://dx.doi.org/10.14359/18770

${ }^{14}$ Langley, R. S. and Heron K. Elastic wave transmission through plate/beam junctions, Journal of Sound and Vibration, 143 (2), 241-253, (1990). https://dx.doi.org/10.1016/0022-460X(90)90953-W

15 Keir, J., Kessissoglou, N. J., and Norwood, C. J. An analytical investigation of single actuator and error sensor control in connected plates, Journal of Sound and Vibration, 271 (3-5), 635-649 (2004). https://dx.doi.org/10.1016/S0022-460X(03)00285-2

${ }^{16}$ Mitrou, G., Ferguson, N., and Renno, J. Wave transmission through two-dimensional structures by the hybrid FE/WFE approach, Journal of Sound and Vibration, 389, 484-501, (2017). https://dx.doi.org/10.1016/j.jsv.2016.09.032

17 Mace, B. R. Periodically stiffened fluid-loaded plates, I: response to convected harmonic pressure and free wave propagation, Journal of Sound and Vibration, 73 (4), 473-486, (1980). https://dx.doi.org/10.1016/0022-460X(80)90662-8

${ }^{18}$ Mace, B. R. Periodically stiffened fluid-loaded plates, II: response to line and point forces, Journal of Sound and Vibration, 73 (4), 487-504, (1980). https://dx.doi.org/10.1016/0022-460X(80)90663-X

${ }^{19}$ Lin, G. G. and Hayek, S. I. Acoustic radiation from point excited rib-reinforced plate, The Journal of the Acoustical Society of America, 62 (1), 72-83, (1977). https://dx.doi.org/10.1121/1.381506

${ }^{20}$ Hull, A. J. and Welch, J. R. Elastic response of an acoustic coating in a rib-stiffened plate, Journal of Sound and Vibration, 329 (20), 4192-4211, (2010). https://dx.doi.org/10.1016/j.jsv.2010.04.012

21 Cauchy, A. L. On the pressure or tension in a solid body, Exercices de Mathématiques, 2, 42-56, (1827).

${ }^{22}$ Love, A. E. H. The small free vibrations and deformation of a thin elastic shell, Philosophical Transactions of the Royal Society of London (A), 179, 491-549, (1888). https://dx.doi.org/10.1098/rsta.1888.0016

23 Soedel, W. Vibrations of Shells and Plates, Third Edition, Revised and Expanded, Marcel Dekker, Inc., New York, (2004).

${ }^{24}$ Graff, K. F. Wave Motion in Elastic Solids, Dover Publications, Inc., New York, (1975).

25 Leissa, A. NASA Technical Report SP-160, Vibration of Plates, Washington, DC, (1969). 


\section{APPENDIX A-MATRIX ENTRIES}

The nonzero entries to the $[\mathbf{A}]$ matrix in Eq. (43) are

$$
a_{1,1}=\frac{-E}{1-\nu^{2}} b_{w}\left(\alpha^{2}+\nu k_{y}^{2}\right) \sin \left(\alpha h_{w}\right)
$$

$a_{1,2}=\frac{-E}{1-\nu^{2}} b_{w}\left(\alpha^{2}+\nu k_{y}^{2}\right) \cos \left(\alpha h_{w}\right)$;

$a_{1,3}=\frac{E}{1+\nu} b_{w} \beta i k_{y} \cos \left(\beta h_{w}\right)$;

$a_{1,4}=\frac{-E}{1+\nu} b_{w} \beta i k_{y} \sin \left(\beta h_{w}\right)$;

$a_{2,1}=\frac{E}{1+\nu} b_{w} \alpha i k_{y} \cos \left(\alpha h_{w}\right)$;

$a_{2,2}=\frac{-E}{1+\nu} b_{w} \alpha i k_{y} \sin \left(\alpha h_{w}\right)$;

$a_{2,3}=\frac{E}{2(1+\nu)} b_{w}\left(\beta^{2}-k_{y}^{2}\right) \sin \left(\beta h_{w}\right)$;

$a_{2,4}=\frac{E}{2(1+\nu)} b_{w}\left(\beta^{2}-k_{y}^{2}\right) \cos \left(\beta h_{w}\right) ;$

$a_{3,5}=D_{w} \xi_{1}\left(\xi_{1}^{2}-\nu k_{y}^{2}+2 k_{y}^{2}\right) \cos \left(\xi_{1} h_{w}\right) ;$

$a_{3,6}=-D_{w} \xi_{1}\left(\xi_{1}^{2}-\nu k_{y}^{2}+2 k_{y}^{2}\right) \sin \left(\xi_{1} h_{w}\right)$;

$a_{3,7}=-D_{w} \xi_{2}\left(\xi_{2}^{2}+\nu k_{y}^{2}-2 k_{y}^{2}\right) \cosh \left(\xi_{2} h_{w}\right)$

$a_{3,8}=-D_{w} \xi_{2}\left(\xi_{2}^{2}+\nu k_{y}^{2}-2 k_{y}^{2}\right) \sinh \left(\xi_{2} h_{w}\right)$;

$a_{4,5}=D_{w}\left(\xi_{1}^{2}+\nu k_{y}^{2}\right) \sin \left(\xi_{1} h_{w}\right)$;

$a_{4,6}=D_{w}\left(\xi_{1}^{2}+\nu k_{y}^{2}\right) \cos \left(\xi_{1} h_{w}\right)$;

$a_{4,7}=D_{w}\left(-\xi_{2}^{2}+\nu k_{y}^{2}\right) \sinh \left(\xi_{2} h_{w}\right)$;

$a_{4,8}=D_{w}\left(-\xi_{2}^{2}+\nu k_{y}^{2}\right) \cosh \left(\xi_{2} h_{w}\right)$;

$a_{5,9}=\frac{-E}{1-\nu^{2}} h_{f}\left(\alpha^{2}+\nu k_{y}^{2}\right) \sin (\alpha a)$;

$a_{5,10}=\frac{-E}{1-\nu^{2}} h_{f}\left(\alpha^{2}+\nu k_{y}^{2}\right) \cos (\alpha a)$;

$a_{5,11}=\frac{E}{1+\nu} h_{f} \beta i k_{y} \cos (\beta a)$;

$a_{5,12}=\frac{-E}{1+\nu} h_{f} \beta i k_{y} \sin (\beta a)$;

$a_{6,9}=\frac{E}{1+\nu} h_{f} \alpha i k_{y} \cos (\alpha a)$;

$a_{6,10}=\frac{-E}{1+\nu} h_{f} \alpha i k_{y} \sin (\alpha a)$;

$a_{6,11}=\frac{E}{2(1+\nu)} h_{f}\left(\beta^{2}-k_{y}^{2}\right) \sin (\beta a) ;$

$a_{6,12}=\frac{E}{2(1+\nu)} h_{f}\left(\beta^{2}-k_{y}^{2}\right) \cos (\beta a) ;$

$a_{7,13}=D_{f l} \xi_{3}\left(\xi_{3}^{2}-\nu k_{y}^{2}+2 k_{y}^{2}\right) \cos \left(\xi_{3} a\right) ;$

$a_{7,14}=-D_{f l} \xi_{3}\left(\xi_{3}^{2}-\nu k_{y}^{2}+2 k_{y}^{2}\right) \sin \left(\xi_{3} a\right) ;$

$a_{7,15}=-D_{f l} \xi_{4}\left(\xi_{4}^{2}+\nu k_{y}^{2}-2 k_{y}^{2}\right) \cosh \left(\xi_{4} a\right)$;

$a_{7,16}=-D_{f l} \xi_{4}\left(\xi_{4}^{2}+\nu k_{y}^{2}-2 k_{y}^{2}\right) \sinh \left(\xi_{4} a\right)$

$a_{8,13}=D_{f l}\left(\xi_{3}^{2}+\nu k_{y}^{2}\right) \sin \left(\xi_{3} a\right) ;$

$a_{8,14}=D_{f l}\left(\xi_{3}^{2}+\nu k_{y}^{2}\right) \cos \left(\xi_{3} a\right) ;$

$a_{8,15}=D_{f l}\left(-\xi_{4}^{2}+\nu k_{y}^{2}\right) \sinh \left(\xi_{4} a\right)$;

$a_{8,16}=D_{f l}\left(-\xi_{4}^{2}+\nu k_{y}^{2}\right) \cosh \left(\xi_{4} a\right)$;
$a_{9,17}=\frac{-E}{1-\nu^{2}} h_{f}\left(\alpha^{2}+\nu k_{y}^{2}\right) \sin (\alpha b) ;$

(A.33)

$a_{9,18}=\frac{-E}{1-\nu^{2}} h_{f}\left(\alpha^{2}+\nu k_{y}^{2}\right) \cos (\alpha b) ;$

(A.34)

$a_{9,19}=\frac{E}{1+\nu} h_{f} \beta i k_{y} \cos (\beta b) ;$

$a_{9,20}=\frac{-E}{1+\nu} h_{f} \beta i k_{y} \sin (\beta b) ;$

$a_{10,17}=\frac{E}{1+\nu} h_{f} \alpha i k_{y} \cos (\alpha b)$;

$a_{10,18}=\frac{-E}{1+\nu} h_{f} \alpha i k_{y} \sin (\alpha b) ;$

$a_{10,19}=\frac{E}{2(1+\nu)} h_{f}\left(\beta^{2}-k_{y}^{2}\right) \sin (\beta b) ;$

$a_{10,20}=\frac{E}{2(1+\nu)} h_{f}\left(\beta^{2}-k_{y}^{2}\right) \cos (\beta b) ;$

(A.40)

$a_{11,21}=D_{f r} \xi_{3}\left(\xi_{3}^{2}-\nu k_{y}^{2}+2 k_{y}^{2}\right) \cos \left(\xi_{3} b\right) ;$

$a_{11,22}=-D_{f r} \xi_{3}\left(\xi_{3}^{2}-\nu k_{y}^{2}+2 k_{y}^{2}\right) \sin \left(\xi_{3} b\right)$;

$a_{11,23}=-D_{f r} \xi_{4}\left(\xi_{4}^{2}+\nu k_{y}^{2}-2 k_{y}^{2}\right) \cosh \left(\xi_{4} b\right)$;

$a_{11,24}=-D_{f r} \xi_{4}\left(\xi_{4}^{2}+\nu k_{y}^{2}-2 k_{y}^{2}\right) \sinh \left(\xi_{4} b\right)$;

$a_{12,21}=D_{f r}\left(\xi_{3}^{2}+\nu k_{y}^{2}\right) \sin \left(\xi_{3} b\right)$;

$a_{12,22}=D_{f r}\left(\xi_{3}^{2}+\nu k_{y}^{2}\right) \cos \left(\xi_{3} b\right)$;

$a_{12,23}=D_{f r}\left(-\xi_{4}^{2}+\nu k_{y}^{2}\right) \sinh \left(\xi_{4} b\right)$;

$a_{12,24}=D_{f r}\left(-\xi_{4}^{2}+\nu k_{y}^{2}\right) \cosh \left(\xi_{4} b\right)$;

$a_{13,2}=\frac{-E}{1-\nu^{2}} b_{w}\left(\alpha^{2}+\nu k_{y}^{2}\right)$;

$a_{13,3}=\frac{E}{1+\nu} b_{w} \beta i k_{y} ;$

$a_{13,13}=-D_{f l} \xi_{3}\left(\xi_{3}^{2}-\nu k_{y}^{2}+2 k_{y}^{2}\right)$;

$a_{13,15}=D_{f l} \xi_{4}\left(\xi_{4}^{2}+\nu k_{y}^{2}-2 k_{y}^{2}\right)$;

$a_{13,21}=D_{f r} \xi_{3}\left(\xi_{3}^{2}-\nu k_{y}^{2}+2 k_{y}^{2}\right)$;

$a_{13,15}=-D_{f r} \xi_{4}\left(\xi_{4}^{2}+\nu k_{y}^{2}-2 k_{y}^{2}\right)$;

$a_{14,5}=D_{w} \xi_{1}\left(\xi_{1}^{2}-\nu k_{y}^{2}+2 k_{y}^{2}\right) ;$

$a_{14,7}=-D_{w} \xi_{2}\left(\xi_{2}^{2}-\nu k_{y}^{2}+2 k_{y}^{2}\right)$;

$a_{14,10}=\frac{-E}{1-\nu^{2}} h_{f}\left(\alpha^{2}+\nu k_{y}^{2}\right)$;

$a_{14,11}=\frac{E}{1+\nu} h_{f} \beta i k_{y} ;$

$a_{14,18}=\frac{E}{1-\nu^{2}} h_{f}\left(\alpha^{2}+\nu k_{y}^{2}\right)$;

$a_{14,19}=\frac{-E}{1+\nu} h_{f} \beta i k_{y}$;

$a_{15,1}=\frac{E}{1+\nu} b_{w} \alpha i k_{y}$;

$a_{15,4}=\frac{E}{2(1+\nu)} b_{w}\left(\beta^{2}-k_{y}^{2}\right) ;$

$a_{15,9}=\frac{-E}{1+\nu} h_{f} \alpha i k_{y}$;

$a_{15,12}=\frac{-E}{2(1+\nu)} h_{f}\left(\beta^{2}-k_{y}^{2}\right)$;

$a_{15,17}=\frac{E}{1+\nu} h_{f} \alpha i k_{y}$;

(A.65) 


$$
\begin{aligned}
& a_{15,20}=\frac{E}{2(1+\nu)} h_{f}\left(\beta^{2}-k_{y}^{2}\right) ; \\
& a_{16,6}=D_{w}\left(\xi_{1}^{2}+\nu k_{y}^{2}\right) ; \\
& a_{16,8}=D_{w}\left(-\xi_{2}^{2}+\nu k_{y}^{2}\right) ; \\
& a_{16,14}=-D_{f l}\left(\xi_{3}^{2}+\nu k_{y}^{2}\right) \text {; } \\
& a_{16,16}=-D_{f l}\left(-\xi_{4}^{2}+\nu k_{y}^{2}\right) ; \\
& a_{16,22}=D_{f r}\left(\xi_{3}^{2}+\nu k_{y}^{2}\right) \text {; } \\
& a_{16,24}=D_{f r}\left(-\xi_{4}^{2}+\nu k_{y}^{2}\right) \text {; } \\
& a_{17,1}=\alpha \text {; } \\
& a_{17,4}=i k_{y} \text {; } \\
& a_{17,14}=-1 \text {; } \\
& a_{17,16}=-1 \text {; } \\
& a_{18,1}=\alpha \text {; } \\
& a_{18,4}=i k_{y} \text {; } \\
& a_{18,22}=-1 \text {; } \\
& a_{18,24}=-1 \text {; } \\
& a_{19,6}=1 \text {; } \\
& a_{19,8}=1 \text {; } \\
& a_{19,9}=\alpha \text {; } \\
& a_{19,12}=i k_{y} \text {; } \\
& a_{20,6}=1 \text {; } \\
& a_{20,8}=1 \text {; } \\
& a_{20,17}=\alpha \text {; } \\
& a_{20,20}=i k_{y} \text {; } \\
& a_{21,2}=i k_{y} \text {; } \\
& a_{21,3}=-\beta \text {; } \\
& a_{21,10}=-i k_{y} \text {; } \\
& a_{21,11}=\beta \text {; } \\
& a_{22,2}=i k_{y} \text {; } \\
& a_{22,3}=-\beta \text {; } \\
& a_{22,18}=-i k_{y} \text {; } \\
& a_{22,19}=\beta \text {; } \\
& a_{23,5}=\xi_{1} \text {; } \\
& a_{23,7}=\xi_{2} \text {; } \\
& a_{23,13}=-\xi_{3} \text {; } \\
& a_{23,15}=-\xi_{4} \text {; } \\
& a_{24,5}=\xi_{1} \text {; } \\
& a_{24,7}=\xi_{2} \text {; } \\
& a_{24,21}=-\xi_{3} \text {; }
\end{aligned}
$$

and

$$
a_{24,23}=-\xi_{4}
$$

\section{APPENDIX B-BICKFORD BEAM}

The mathematical model of a Bickford beam begins with the assumption that the beam has an in-plane displacement distribution that is a third order function, with respect to the depth of the beam. The displacement field of this beam is written as

$$
u(y, z, t)=z \phi(y, t)-\frac{4 z^{3}}{3 h^{2}}\left(\phi(y, t)+\frac{\partial w_{0}(y, t)}{\partial y}\right)
$$

and

$$
w(y, z, t)=w_{0}(y, t)
$$

where $y$ is the coordinate along the axis of the beam, $z$ is the coordinate transverse to the beam, with $z=0$ located at the neutral axis, $t$ is time, $u(y, z, t)$ is the displacement field in the axial direction, $w(y, z, t)$ is the displacement field in the transverse direction, $\phi(y, t)$ is the rotation of the beam at the neutral axis, and $h$ is the total height of the beam. As the beam is infinitely long, the normal displacement and rotation of the beam are written as exponential functions in space and time as

$$
w_{0}(y, t)=W \exp \left(i k_{y} y\right) \exp (i \omega t)
$$

and

$$
\phi(y, t)=X \exp \left(i k_{y} y\right) \exp (i \omega t) ;
$$

where $W$ and $X$ are unknown wave propagation coefficients. Using the principal of virtual displacement, the wave propagation coefficients can be determined with

$$
\left[\begin{array}{ll}
a_{11} & a_{12} \\
a_{21} & a_{22}
\end{array}\right]\left\{\begin{array}{l}
X \\
W
\end{array}\right\}=\left\{\begin{array}{c}
0 \\
P_{0}
\end{array}\right\} .
$$

For a rectangular beam with dimensions base $b$ and height $h$, the matrix entries are

$$
\begin{aligned}
& a_{11}=\frac{-17 \rho \omega^{2} b h^{3}}{315}+\frac{17 k_{y}^{2} E b h^{3}}{315}+\frac{8 G b h}{15} ; \\
& a_{12}=\frac{4 i k_{y} \rho \omega^{2} b h^{3}}{315}+\frac{-4 i k_{y}^{3} E b h^{3}}{315}+\frac{8 i k_{y} G b h}{15} ; \\
& a_{21}=\frac{4 i k_{y} \rho \omega^{2} b h^{3}}{315}+\frac{-4 i k_{y}^{3} E b h^{3}}{315}+\frac{8 i k_{y} G b h}{15} ;
\end{aligned}
$$

and

$$
a_{22}=\frac{k_{y}^{2} \rho \omega^{2} b h^{3}}{252}+\rho \omega^{2} b h+\frac{-k_{y}^{4} E b h^{3}}{252}+\frac{-8 k_{y}^{2} G b h}{15} .
$$

For a T-shaped beam, these entries are much longer and are not included for brevity. They are, however, calculated in the exact same manner as Eqs. (B.1)-(B.5). The normal displacement field can be determined from Eq. (B.5) and is

$$
W=\frac{a_{11} P_{0}}{a_{11} a_{22}-a_{12} a_{21}} .
$$

